\title{
Fast-slow mode coupling instability for coasting beams in the presence of detuning impedance
}

\author{
N. Biancacci® ${ }^{1}$ E. Métral๑, ${ }^{1}$ and M. Migliorati ${ }^{2}$ \\ ${ }^{1}$ CERN, 1211 Geneva 23, Geneva, Switzerland \\ ${ }^{2}$ University of Rome, "La Sapienza" and INFN - Roma 1, Piazzale Aldo Moro, 5, 00185 Roma, Italy
}

(Received 9 October 2020; accepted 16 December 2020; published 31 December 2020)

\begin{abstract}
A destabilizing effect of the detuning impedance has been recently observed in simulations of the CERN Proton Synchrotron (PS) at the injection energy: while without the detuning impedance the instability is faster in the vertical plane as expected (due to the elliptical shape of the vacuum chamber), with detuning impedance the instability appears to be faster in the horizontal plane. In order to understand the detuning impedance destabilizing effect, we study the collective behavior for the simpler case of a coasting beam with PS-like parameters and a simplified impedance model. The analysis, carried out from both numerical and theoretical points of view, highlights a new destabilizing mechanism related to the coupling of slow and fast waves.
\end{abstract}

DOI: 10.1103/PhysRevAccelBeams.23.124402

\section{INTRODUCTION}

The CERN Proton Synchrotron (PS) provides high intensity proton bunches within the injector chain to the Large Hadron Collider (LHC). The machine and beam parameters have been recently pushed in order to meet the challenging goals of the LHC injectors upgrade (LIU) project $[1,2]$ started in 2010. For the future operation at higher intensities and beam brightness required by the LIU project, it is of fundamental importance to detect and cure the observed machine instabilities along the LHC beam production cycles. Headtail horizontal instabilities have been observed, measured and systematically characterized versus chromaticity at the injection kinetic energy of $1.4 \mathrm{GeV}$ with a bunched beam in [3]. The analysis of the instability based on Sacherer's theory [4] was found to be in good agreement with measurements concerning the headtail mode number [3]. One of the possible explanations for the horizontal plane to be more unstable than the vertical one (which is not expected for a flat vacuum chamber, as, in first approximation, the one of the PS) was a different chromaticity between the two planes. The mode pattern and behavior with chromaticity was also studied with a macroparticle code [5]: HEADTAIL [6] simulations were performed scaling the PS resistive wall wakefield to reduce the simulation time and without accounting for the effect of the direct space charge and detuning

Published by the American Physical Society under the terms of the Creative Commons Attribution 4.0 International license. Further distribution of this work must maintain attribution to the author(s) and the published article's title, journal citation, and DOI. impedance. The simulation results confirmed the measurements [3]. Recently, the instability was simulated with the PyHEADTAIL code [7] by using the PS impedance model developed in [8,9] and it revealed a destabilising effect of the detuning impedance for some chromaticities in the horizontal plane, otherwise more stable than the vertical plane in the presence of only driving impedance [10].

In order to understand the instability mechanism and to investigate why the horizontal plane is destabilised by the detuning impedance, a series of studies was recently performed at CERN. The effect of the detuning impedance was investigated with a two-particle model [11], with systematic bunched beam PyHEADTAIL simulations [12], with the eDELPHI Vlasov solver [13], and with the circulant matrix formalism [14,15].

In this context, we analyze the effect of the detuning impedance on a coasting beam with PS parameters and a simple impedance model of two resistive parallel plates. On one hand, this approach offers the advantage of a simpler collective effects treatment to investigate if a destabilising effect of the detuning impedance can already be observed in the case of a coasting-beam (with the simplified impedance model) and for zero chromaticity. On the other hand, it does not aim to directly explain the PS instability, even if it shows that indeed a destabilizing effect is present also in this case.

The theoretical background for the study of coasting beam collective effects is described in Sec. II. Simulations accounting for the driving impedance only will be compared with the classical theory developed in [16-18]. In Sec. III the theory is extended in order to include the detuning impedance (not accounted for in the classical treatment [16-18]). These modifications are essential 
ingredients to be able to reproduce simulations performed in presence of both driving and detuning impedances. In Sec. IV the theory is further extended to account possible couplings between fast and slow coasting beam betatron waves: the fast-slow mode coupling instability is described and benchmarked with PyHEADTAIL simulations. Section V summarizes the results described in the previous sections.

\section{TRANSVERSE COASTING BEAM INSTABILITY SIMULATIONS WITH DRIVING IMPEDANCE}

In this section we introduce the theory and the simulations of coasting beam transverse instabilities following the approach of [17]. This will be the starting point for the following developments presented in Sec. III to correctly account for the additional effect of the detuning impedance.

Let us consider a coasting proton beam of charge $q N$ with $q$ the elementary charge and $N$ the number of charges in the beam. The line density distribution $\rho(s)$ is supposed to be constant and equal to $\rho(s)=q N / C$ where $C$ is the accelerator circumference. We consider a transverse perturbation $d(s, t)$ as a function of space $s$ and time $t$ with the form of

$$
d(s, t)=\Delta e^{j(\Omega t-n s / R)},
$$

where $R=C / 2 \pi$ is the average radius of the machine, $n$ an arbitrary integer number and $\Omega$ is the angular frequency of the perturbation. Equation (1) describes a wave of $n$ periods within the accelerator circumference, oscillating with the collective frequency $\Omega$.

The dipole moment of the coasting beam is given by

$$
D(s, t)=d(s, t) \rho(s)=\frac{q N \Delta}{C} e^{j(\Omega t-n s / R)} .
$$

Given a beam circulating with velocity $v=\beta c$ with $\beta$ the relativistic factor and $c$ the speed of light, a particle in $s_{0}$ at $t=0$ will move according to $D\left(s_{0}+v t, t\right)$.

Let us now consider a particle performing betatron oscillations, for example, in the vertical plane $y$, and perturbed by the wakefields produced by the perturbation. The equation of motion can be written as [17]

$$
\ddot{y}(s, t)+\omega_{\beta}^{2} y(s, t)=\frac{\left\langle F_{y}\right\rangle(s, t)}{\gamma m_{0}},
$$

where $\omega_{\beta}=Q_{y} \omega_{0}$ is the vertical betatron angular frequency with $Q_{y}$ the machine vertical tune and $\omega_{0}$ the revolution angular frequency, $\gamma$ is the relativistic Lorentz factor, $m_{0}$ the rest mass of the particle, $\left\langle F_{y}\right\rangle(s, t)$ is the average force acting on the particle motion, and $\dot{y}$ means derivation of $y$ with respect to $t$.

In the frame of wakefields, the force induced by a source particle on a following test particle at distance $z>0$, can be expressed as

$$
F_{y}=-\frac{q^{2}}{C} W_{y}(\bar{y}, y)
$$

where $\bar{y}$ and $y$ respectively indicate the displacement of the source and test charges. The Taylor expansion around the center of the beam vacuum chamber gives

$$
F_{y}=-\frac{q^{2}}{C}\left(\left.\frac{\partial W_{y}}{\partial \bar{y}}\right|_{y=0} \bar{y}+\left.\frac{\partial W_{y}}{\partial y}\right|_{\bar{y}=0} y\right),
$$

where we neglected the constant term for simplicity. We define $W_{y}^{\text {driv }}=\partial W_{y} /\left.\partial \bar{y}\right|_{y=0}$ the driving wake function and $W_{y}^{\text {det }}=\partial W_{y} /\left.\partial y\right|_{\bar{y}=0}$ the detuning wake function [19] in units of $\mathrm{V} /(\mathrm{C} \mathrm{m})$. We introduce as well the driving and detuning impedances as the Fourier transform of their corresponding wake functions:

$$
Z_{y}^{\mathrm{driv} / \mathrm{det}}(\omega)=-j \int_{0}^{+\infty} W_{y}^{\mathrm{driv} / \mathrm{det}}(z) e^{j \omega z / v} \mathrm{~d} z / v .
$$

Considering, for the moment, the absence of detuning wake, and the perturbation defined in Eq. (2), the total force acting on a charge at a fixed point in the accelerator can be rewritten as

$$
\begin{aligned}
\left\langle F_{y}^{\text {driv }}\right\rangle(s, t) & =-\frac{q}{C} \int_{t}^{\infty} W_{y}^{\text {driv }}\left(v t^{\prime}-v t\right) D\left(s, t^{\prime}\right) v \mathrm{~d} t^{\prime} \\
& =-\frac{q^{2} N \Delta}{C^{2}} e^{-j n s / R} \int_{t}^{\infty} W_{y}^{\text {driv }}\left(v t^{\prime}-v t\right) e^{j \Omega t^{\prime}} v \mathrm{~d} t^{\prime} \\
& =-\frac{q^{2} N v \Delta}{C^{2}} e^{-j n s / R} e^{j \Omega t} \int_{0}^{\infty} W_{y}^{\text {driv }}(z) e^{j \Omega z / v} \mathrm{~d} z / v .
\end{aligned}
$$

Using the impedance definition of Eq. (6) we have

$$
\left\langle F_{y}^{\mathrm{driv}}\right\rangle(s, t)=-\frac{q v}{C} D(s, t) j Z_{y}^{\mathrm{driv}}(\Omega) .
$$

This force acts on a charge at a fixed location into an accelerator. A particle that passes in $s=s_{0}$ at $t=0$ will be affected by a force at the time $t$ equal to $\left\langle F_{y}^{\text {driv }}\right\rangle(s, t)$ where $s=s_{0}+R \omega_{0} t=s_{0}+v t$. Equation (3) can then be written as

$\ddot{y}(s, t)+\omega_{\beta}^{2} y(s, t)=-\frac{q v}{\gamma m_{0} C} D\left(s_{0}+v t, t\right) j Z_{y}^{\text {driv }}(\Omega)$.

Assuming $y(s, t)=A e^{\left.j\left(\left(\Omega-n \omega_{0}\right) t-n s_{0} / R\right)\right)}$ as trial particular solution of the differential equation, we can find the constant $A$ substituting the expression into Eq. (9) and getting

$$
y(s, t)=-\frac{q^{2} v N}{\gamma m_{0} C^{2}} \Delta j Z_{y}^{\text {driv }}(\Omega) \frac{e^{j(\Omega t-n s / R)}}{\omega_{\beta}^{2}-\left(\Omega-n \omega_{0}\right)^{2}} .
$$


To be self-consistent with the initial assumption of coherent steady-state oscillations of Eq. (2), $y(s, t)$ is also equal to

$$
y(s, t)=\Delta e^{j(\Omega t-n s / R)} .
$$

From Eqs. (11) and (10), we get the dispersion relation

$$
1=-\frac{q^{2} v N}{\gamma m_{0} C^{2}} j Z_{y}^{\text {driv }}(\Omega) \frac{1}{\omega_{\beta}^{2}-\left(\Omega-n \omega_{0}\right)^{2}} .
$$

Let us now solve for $\Omega$. We rewrite Eq. (12) as

$$
\omega_{\beta}^{2}-\left(\Omega-n \omega_{0}\right)^{2}=-\frac{q^{2} v N}{\gamma m_{0} C^{2}} j Z_{y}^{\text {driv }}(\Omega),
$$

from which, defining $\Omega=n \omega_{0}-\omega_{\beta}+\Delta \Omega_{n}$, and assuming $\Delta \Omega_{n} \ll n \omega_{0}-\omega_{\beta}$, we have

$$
\Delta \Omega_{n}^{\text {driv }}=-\frac{q^{2} v N}{2 \gamma m_{0} C^{2} \omega_{\beta}} j Z_{y}^{\text {driv }}\left[\left(n-Q_{y}\right) \omega_{0}\right]
$$

The rise time, in number of turns, associated to each line is defined as $\tau_{n}=-1 / \operatorname{Im}\left(\Delta \Omega_{n}^{\text {driv }}\right) / T_{0}$ and we define the normalized frequency shift as $\operatorname{Re}\left(\Delta \Omega_{n}^{\text {driv }}\right) / \omega_{0}$.

Having chosen $n$ as an integer number, the solutions with $n-Q_{y}>0$ will sample $\operatorname{Re}\left(Z_{y}^{\text {driv }}\right)>0$ and the waves are therefore unstable (so called slow waves) with positive rise time, while solutions with $n-Q_{y}<0$ will sample $\operatorname{Re}\left(Z_{y}^{\text {driv }}\right)<0$ and the waves are stable (so called fast waves) with negative rise time.

The PyHEADTAIL code was originally conceived to simulate the collective effects of bunched beams only [6]. We have introduced some modifications to adapt the code to simulate coasting beams: the longitudinal charge density covers the full machine circumference, a simple drift replaces the rf focusing, the wake interaction is computed with the inverse Fourier transform of the product between the impedance and the charge transverse dipole distribution.

In order to benchmark the new PyHEADTAIL code with the complex mode shift of Eq. (14) we assumed a uniform longitudinal charge distribution with neither momentum spread nor chromaticity.

In the frame of this study, we consider the resistive wall impedance of a flat beam pipe with PS-like parameters: two parallel plates of stainless steel (electrical resistivity of $7.2 \times 10^{-7} \Omega \mathrm{m}$ ) with $27.5 \mathrm{~mm}$ half gap, infinitely thick and $628.32 \mathrm{~m}$ long. The impedance is computed with the ImpedanceWake2D code [20,21].

Figure 1 shows the computed driving and detuning impedances: as expected the vertical detuning impedance is half the driving one in the same plane, while the horizontal detuning impedance is opposite in sign to the driving one in the same plane; the driving horizontal is half

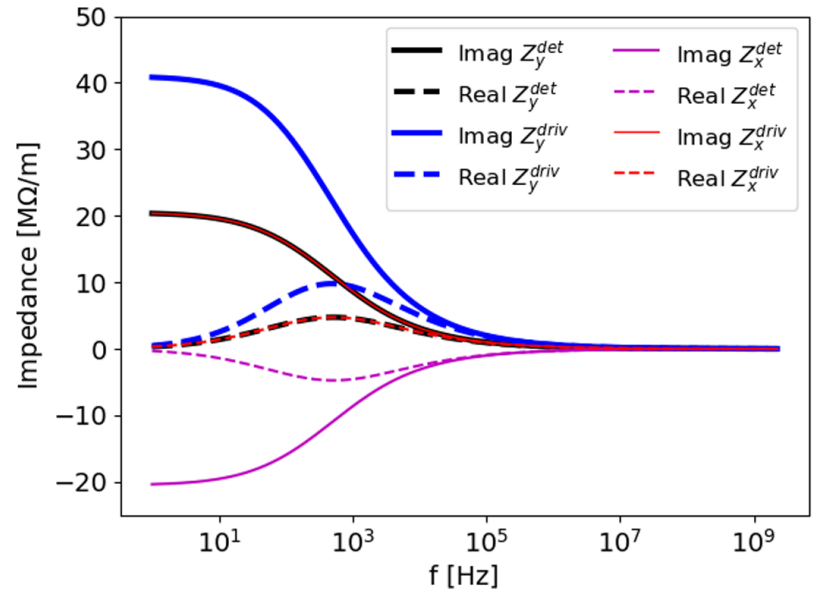

FIG. 1. Vertical driving and detuning impedances (respectively in blue and black), and horizontal driving and detuning impedances (in red and magenta) for a flat pipe made of two parallel plates of stainless steel (electrical resistivity of $7.2 \times 10^{-7} \Omega \mathrm{m}$, $27.5 \mathrm{~mm}$ half gap, infinite thickness, $628.32 \mathrm{~m} \mathrm{long}$ ).

the vertical one [22]. More accurate models for the PS transverse impedance are available [9,23-25] but beyond the scope of this work. It is important to notice the inductive bypass effect toward low frequencies [26,27], at which the effect of the image charges dominates the imaginary part of the impedances.

For the simulation, we assume equal working points in the horizontal and vertical planes $Q_{x, y}=6.4$ but no coupling between the two planes. The beam is approximated by 50000 macroparticles, sliced with 128 bins and tracked for 2000 turns with variable intensity ranging from $1 \times 10^{13}$ to $1 \times 10^{14}$ charges. Figure 2 shows the amplitude spectrum of the vertical plane as a function of the number of turns: since the coasting beam spectrum samples the impedance in the classical thick wall regime, i.e., a

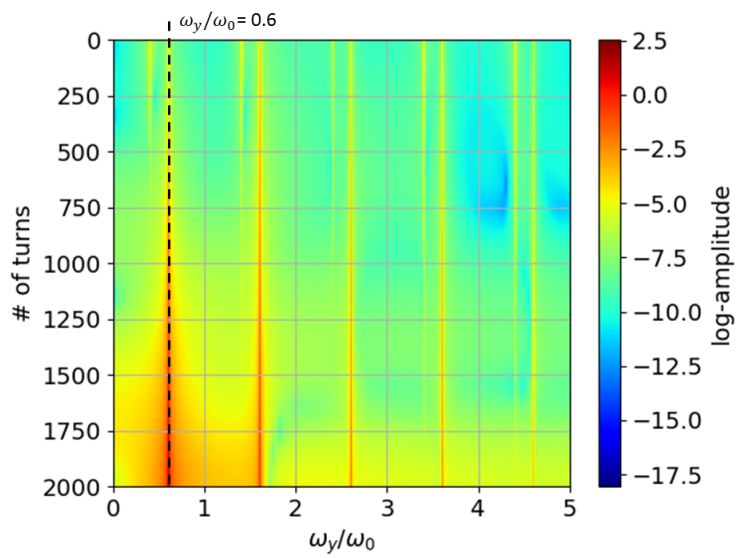

FIG. 2. Amplitude spectrogram of the instability simulated with PyHEADTAIL accounting for the driving impedance only at the intensity of $1 \times 10^{13}$ charges. The most unstable line corresponds to the slow wave $n=7$, for which $\left(n-Q_{y}\right)=0.6$. 

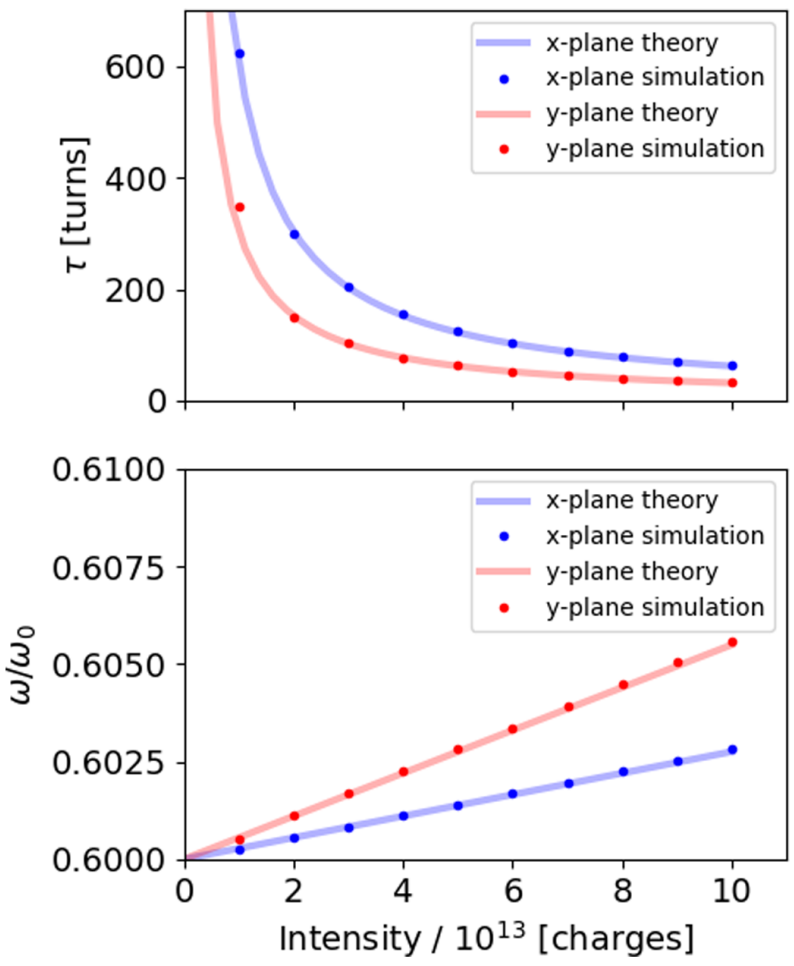

FIG. 3. PyHEADTAIL simulations (with dots) compared to theory [16] for driving impedance only (with full lines) for the horizontal (blue) and the vertical (red) planes. The rise time of the most unstable mode is shown at the top while the normalized frequency shift is shown at the bottom.

monotonically decreasing real part versus frequency, the lowest frequency slow wave corresponding to $n-Q_{y}=0.6$ is the most unstable.

Figure 3 shows the rise time of the instability (at the top) and the normalized frequency shift of the most unstable mode (at the bottom), compared to Eq. (14). The agreement is excellent and it can be seen that, as expected, for all the intensities the vertical plane is more unstable than the horizontal one. It is important to notice that the resolution on the normalized frequency shift has been improved over the classical FFT algorithm by using a harmonic analysis method based on iterative frequency interpolation [28].

\section{TRANSVERSE COASTING BEAM INSTABILITY SIMULATIONS WITH BOTH DRIVING AND DETUNING IMPEDANCES}

We now include the effect of the detuning impedance, first analytically following a treatment similar to the one discussed in Sec. II, then with macroparticles simulations.

From Eq. (5), the infinitesimal source charge $\rho\left(s, t^{\prime}\right) v \mathrm{~d} t^{\prime}$ induces a detuning wakefield on the test particle $y(s, t)$ given by

$\mathrm{d} F_{y}^{\operatorname{det}}(s, t)=-\frac{q}{C} y(s, t) W_{y}^{\operatorname{det}}\left(v t^{\prime}-v t\right) \rho\left(s, t^{\prime}\right) v \mathrm{~d} t^{\prime}$.
Integrating over time we get the total force as

$\left\langle F_{y}^{\mathrm{det}}\right\rangle(s, t)=-\frac{q}{C} y(s, t) \int_{t}^{\infty} W_{y}^{\operatorname{det}}\left(v t^{\prime}-v t\right) \rho\left(s, t^{\prime}\right) v \mathrm{~d} t^{\prime}$.

Differently from the driving force, which is produced only by the perturbation, the detuning force depends on the unperturbed longitudinal distribution $\rho(s, t)$. When this is constant, the detuning force simplifies into

$$
\begin{aligned}
\left\langle F_{y}^{\operatorname{det}}\right\rangle(s, t) & =-\frac{q^{2} N}{C^{2}} y(s, t) \int_{t}^{\infty} W_{y}^{\operatorname{det}}\left(v t^{\prime}-v t\right) v \mathrm{~d} t^{\prime} \\
& =-\frac{q^{2} N}{C^{2}} y(s, t) \int_{0}^{\infty} W_{y}^{\operatorname{det}}(z) \mathrm{d} z .
\end{aligned}
$$

From the definition of impedance of Eq. (6) we have

$$
\int_{0}^{\infty} W_{y}^{\operatorname{det}}(z) \mathrm{d} z=j v Z_{y}^{\operatorname{det}}(0) .
$$

In turn the force can be expressed as

$$
\left\langle F_{y}^{\mathrm{det}}\right\rangle(s, t)=-\frac{q^{2} v N}{C^{2}} y(s, t) j Z_{y}^{\mathrm{det}}(0) .
$$

In presence of detuning impedance only, Eq. (3) is written as follows

$$
\ddot{y}(s, t)+\omega_{\beta}^{2} y(s, t)=-\frac{q^{2} v N}{\gamma m_{0} C^{2}} j Z_{y}^{\operatorname{det}}(0) y(s, t) .
$$

Gathering the coefficients of $y(s, t)$, the effect of the detuning impedance is to add an additional tune shift to the bare machine working point. The new working point is defined as $\omega_{\beta}^{\prime}=\omega_{\beta}-\Delta \Omega_{n}^{\mathrm{det}}$. Solving Eq. (20) for this new frequency and neglecting second order terms in $\Delta \Omega_{n}^{\text {det }}$, we have

$$
\Delta \Omega_{n}^{\mathrm{det}}=-\frac{q^{2} v N}{2 \gamma m_{0} C^{2} \omega_{\beta}} j Z_{y}^{\operatorname{det}}(0)
$$

It is interesting to notice that in the specific case of the resistive wall impedance, the thick-wall regime has $1 / \sqrt{\omega}$ frequency dependence [29], i.e., with a singularity at DC, that disappears only if the inductive bypass effect is taken into account $[26,27]$. In other words, it is necessary to have the full/correct detuning impedance description up to DC (this is not the case for the driving impedance as the lowest needed frequency is the one of the lowest unstable mode).

\section{THE FAST-SLOW WAVES COUPLING}

Let us now consider the effect of both driving and detuning impedances. Comparing respectively Eqs. (9), 

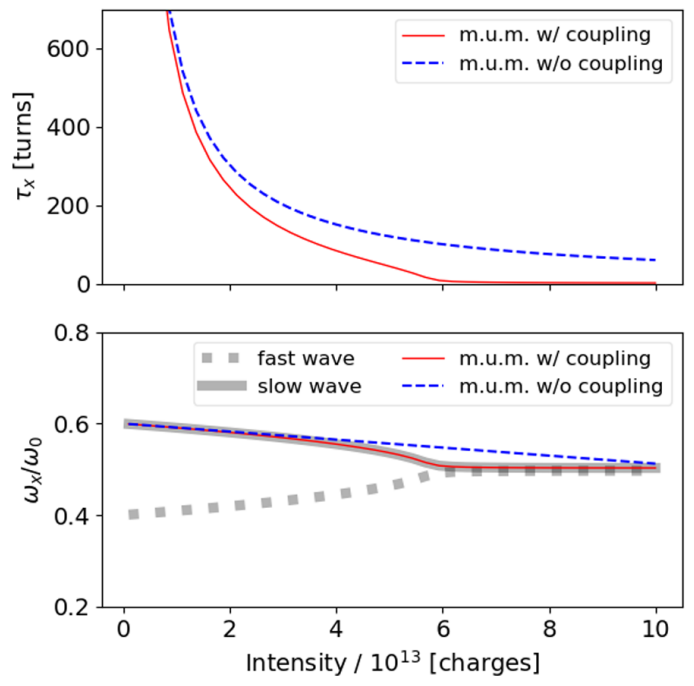

FIG. 4. At the top, the rise time of the most unstable mode (m.u.m.) in the horizontal plane is shown respectively with (red line) and without (dashed blue line) coupling of fast and slow waves. At the bottom, the frequency normalized to the revolution frequency of the m.u.m. with and without coupling is shown together with the normalized frequency shift of the fast (dashed gray line) and slow (full gray line) waves corresponding respectively to $n=6$ and $n=7$.

(11) and (14) for the driving force, and Eqs. (20), (21) for the detuning force, we can write

$$
\ddot{y}(s, t)+\omega_{\beta}^{2} y(s, t)=2 \omega_{\beta}\left(\Delta \Omega_{n}^{\text {driv }}+\Delta \Omega_{n}^{\text {det }}\right) y(s, t) .
$$

In Sec. III we described the effect of the detuning impedance on transverse coasting beam instabilities and the way to correctly account for it from the analytical point of view. As the detuning impedance introduces a large frequency shift due to the sampling at DC, we investigate the possibility of fast-slow waves coupling, so far not investigated in the available literature.

Let us consider two nearby waves, namely a slow wave $y_{1}(s, t)$ corresponding to $n=n_{1}$ and a fast wave $y_{2}(s, t)$ corresponding to $n=n_{2}$. Dropping the dependence on $s$ and $t$, the coupled system can be written as

$$
\left\{\begin{array}{l}
\ddot{y_{1}}+\omega_{\beta}^{2} y_{1}=2 \omega_{\beta} \Delta \Omega_{n_{1}}^{\mathrm{tot}} y_{1}+2 \omega_{\beta} \Delta \Omega_{n_{2}}^{\mathrm{tot}} y_{2} \\
\ddot{y_{2}}+\omega_{\beta}^{2} y_{2}=2 \omega_{\beta} \Delta \Omega_{n_{2}}^{\mathrm{tot}} y_{2}+2 \omega_{\beta} \Delta \Omega_{n_{1}}^{\mathrm{tot}} y_{1}
\end{array}\right.
$$

where $\Delta \Omega_{n_{1}, n_{2}}^{\text {tot }}=\Delta \Omega_{n_{1}, n_{2}}^{\text {driv }}+\Delta \Omega_{n_{1}, n_{2}}^{\text {det }}$. The nontrivial solution of the system represents the solution for the coupled waves. Looking for the solutions in the form of $y_{1,2}=$ $e^{j\left(\Omega-n_{1,2} \omega_{0}\right) t}$ we get the matrix system

$$
\left[\begin{array}{ll}
m_{11} & m_{12} \\
m_{21} & m_{22}
\end{array}\right]\left[\begin{array}{l}
y_{1} \\
y_{2}
\end{array}\right]=\left[\begin{array}{l}
0 \\
0
\end{array}\right]
$$
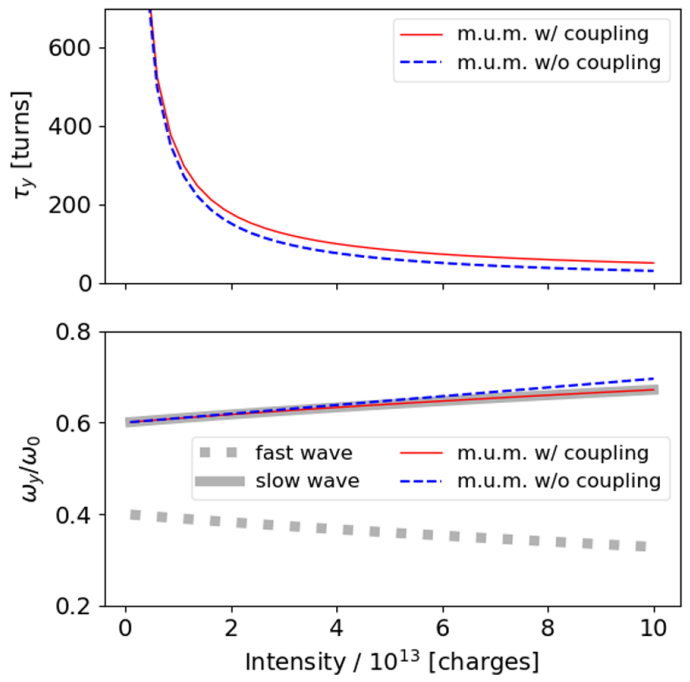

FIG. 5. Same as Fig. 4 for the vertical plane.

with

$$
\begin{aligned}
& m_{11}=\left(\omega_{\beta}+n_{1} \omega_{0}-\Omega\right)\left(\omega_{\beta}-n_{1} \omega_{0}+\Omega\right)-2 \omega_{\beta} \Delta \Omega_{n_{1}}^{\mathrm{tot}}, \\
& m_{12}=-2 \omega_{\beta} \Delta \Omega_{n_{2}}^{\mathrm{tot}}, \\
& m_{21}=-2 \omega_{\beta} \Delta \Omega_{n_{1}}^{\mathrm{tot}} \\
& m_{22}=\left(\omega_{\beta}+n_{2} \omega_{0}-\Omega\right)\left(\omega_{\beta}-n_{2} \omega_{0}+\Omega\right)-2 \omega_{\beta} \Delta \Omega_{n_{2}}^{\mathrm{tot}} .
\end{aligned}
$$

Looking for the nontrivial solutions of the system, for example using [30], we obtain the coupled complex frequency shift of the fast-slow waves.

Figures 4 and 5 show, respectively for the horizontal and vertical plane, the normalized frequency shift and the rise time of the instability accounting for the coupling between adjacent fast and slow waves. As can be seen, the prediction from the coupled waves approach of (24) is approximated by the uncoupled one of Eq. (14) only for intensities below $1 \times 10^{13}$ charges. At $6 \times 10^{13}$ the horizontal plane shows coupling between fast and slow waves, with consequent shorter instability rise time. In the vertical plane, the fast and slow waves are repelling each other, and the difference in complex frequency shift is negligible.

Figure 6 shows the comparison between Eq. (24) and PyHEADTAIL simulations performed with both driving and detuning impedances. The agreement is excellent and shows the importance of considering the coupling of fast and slow waves in order to correctly predict the rise time and frequency shift of the instability. This mechanism is conceptually analogous to the one of a transverse mode coupling instability (TMCI) occurring when bunched beam modes couple with each other [18]. Similarly, coupling occurs between fast and slow waves in coasting beams. 

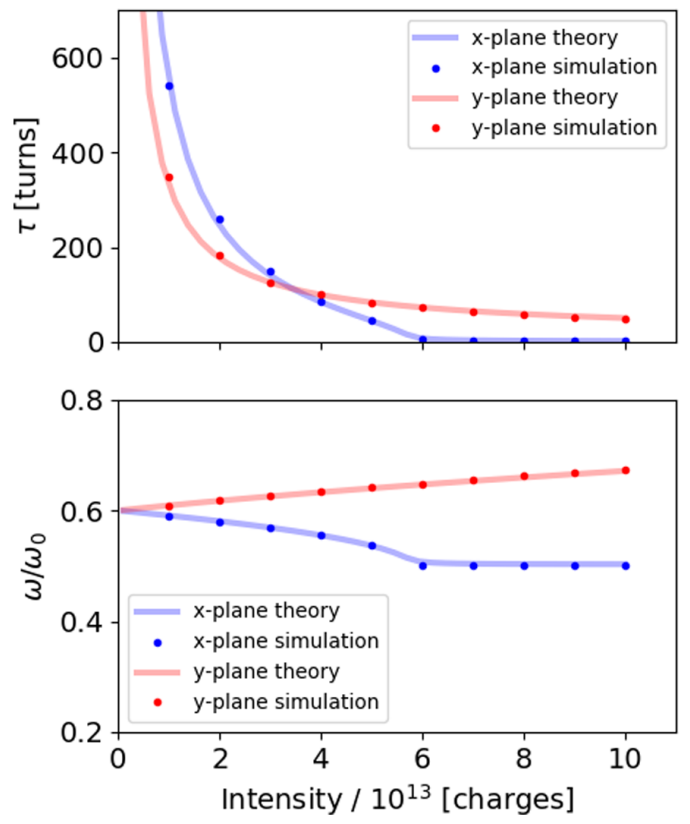

FIG. 6. PyHEADTAIL simulations (with dots) compared to theory accounting for coupling between fast and slow waves (with full lines) for the horizontal (blue) and the vertical (red) planes. The rise time, at the top, and the normalized frequency shift, at the bottom, are shown.

\section{CONCLUSIONS}

In this paper we described the effect of the driving and detuning impedance on transverse coasting beam instabilities.

The PyHEADTAIL code has been extended to include the study of coasting beam collective effects in presence of wakefields. The code was successfully benchmarked with the already developed theory of instabilities induced by the driving impedance.

The effect of the detuning impedance was studied both analytically and numerically: the impedance is sampled at DC for a perfect coasting beam, and can therefore induce significantly larger frequency shift compared to the driving impedance only. This result is presently being investigated following also the Vlasov's formalism and will allow future studies on the effect of a finite momentum spread and chromaticity.

This study revealed the possibility of a new instability mechanism: the coupling between adjacent fast and slow waves. The mechanism has been successfully reproduced with PyHEADTAIL numerical simulations.

This work supports, from a different point of view, the necessity of having a correct description of the inductive bypass impedance of a machine (i.e., a correct description of the impedance down to DC).

\section{ACKNOWLEDGMENTS}

This work was partially supported by the European Commission under the HORIZON 2020 Integrating
Activity project ARIES, Grant agreement No. 730871, by the Conseil Européen pour la Recherche Nucléaire PSLIU project, and by INFN National committee V trough the ARYA project.

[1] R. Garoby et al., Status and plans for the upgrade of the LHC injectors, Report No. CERN-ATS-2013-059, 2013, p. 3, https://cds.cern.ch/record/1566636.

[2] H. Damerau et al., LHC Injectors Upgrade, Technical Design Report, Tech. Report No. CERN-ACC-2014-0337, 2014, http://cds.cern.ch/record/1976692.

[3] R. Cappi, R. Garoby, and E. Métral, Collective effects in the CERN-PS beam for LHC, CERN, Geneva, Tech. Report No. CERN-PS-99-049-CA, 1999, https://cds.cern .ch/record/398765.

[4] F. J. Sacherer, Transverse bunched beam instabilities - Theory, Conf. Proc. C 740502, 347 (1975), http://cds .cern.ch/record/322645.

[5] E. Métral, G. Rumolo, R. Steerenberg, and B. Salvant, Simulation study of the horizontal head tail instability observed at injection of the CERN Proton Synchrotron, in 22nd Particle Accelerator Conference, Albuquerque, NM, USA, edited by C. Petit-Jean-Genaz, pp. 4210-4212, https://doi.org/10.1109/PAC.2007.4439983.

[6] G. Rumolo and F. Zimmermann, Electron cloud simulations: beam instabilities and wakefields, Phys. Rev. Accel. Beams 5, 121002 (2002).

[7] "PyHEADTAIL code repository." https://github.com/ PyCOMPLETE/.

[8] M. Migliorati, S. Persichelli, H. Damerau, S. Gilardoni, S. Hancock, and L. Palumbo, Beam-wall interaction in the cern proton synchrotron for the lhc upgrade, Phys. Rev. Accel. Beams 16, 031001 (2013).

[9] S. Persichelli, M. Migliorati, N. Biancacci, S. Gilardoni, E. Métral, and B. Salvant, Transverse beam coupling impedance of the CERN Proton Synchrotron, Phys. Rev. Accel. Beams 19, 041001 (2016).

[10] M. Migliorati et al., Short update of the PS instability studies at injection, HSC meeting, CERN, Geneva, Switzerland, Jun. 2019, https://indico.cern.ch/event/824835/.

[11] G. Rumolo, Two-particle model including quadrupolar wake fields, in 8th Low Emittance Rings Workshop, INFN-LNF, Frascati, Italy, 2020, https://agenda.infn.it/event/20813/.

[12] N. Chitnis et al., Study of the effect of detuning impedance on the transverse beam dynamics, HSC meeting, CERN, Geneva, Switzerland, Aug. 2020, https://indico.cern.ch/ event/947183/.

[13] G. Iadarola, L. Mether, N. Mounet, and L. Sabato, Linearized method for the study of transverse instabilities driven by electron clouds, Phys. Rev. Accel. Beams 23, 081002 (2020).

[14] E. Métral et al., Intra-bunch motion and TMCI in the presence of detuning impedance, in 8th Low Emittance Rings Workshop, INFN-LNF, Frascati, Italy, 2020, https:// agenda.infn.it/event/20813/.

[15] E. Métral, X. Buffat, and G. Rumolo, Transverse mode-coupling instability in the presence of detuning impedance, Report No. CERN-ACC-NOTE-2020-0019, 
Apr 2020, CERN, Geneva, Switzerland, https://cds.cern .ch/record/2714848.

[16] J. L. Laclare, Coasting beam transverse coherent instabilities, in CERN Accelerator School: Course on General Accelerator Physics, 1992, pp. 385-408, https://cds.cern .ch/record/398298.

[17] K. Y. Ng, Physics of Intensity Dependent Beam Instabilities (World Scientific, Singapore, 2005).

[18] A. Chao, Physics of collective beam instabilities in highenergy accelerators, 1993, https://www.slac.stanford.edu/ achao/wileybook.html.

[19] S. Heifets, A. Wagner, and B. Zotter, Generalized impedances and wakes in asymmetric structures, Report No. SLAC-AP-110, 1, 1998, http://slac.stanford.edu/ pubs/apnotes/slac-ap-110.pdf.

[20] N. Mounet and E. Métral, Electromagnetic fields and beam coupling impedances in a multilayer flat chamber, Dec 2010, https://cds.cern.ch/record/1314524.

[21] ImpedanceWake2D code repository, https://twiki.cern.ch/ twiki/bin/view/ABPComputing/ImpedanceWake2D.

[22] K. Yokoya, Resistive wall impedance of beam pipes of general cross section, Part. Accel. 41, 221 (1993); KEKPreprint-92-196, 1993, http://cds.cern.ch/record/248630/.

[23] S. Persichelli, N. Biancacci, M. Migliorati, L. Palumbo, and V. G. Vaccaro, Electromagnetic fields and Green's functions in elliptical vacuum chambers, Phys. Rev. Accel. Beams 20, 101004 (2017).
[24] M. Migliorati, N. Biancacci, M. R. Masullo, L. Palumbo, and V. G. Vaccaro, Space charge impedance and electromagnetic fields in elliptical vacuum chambers, Phys. Rev. Accel. Beams 21, 124201 (2018).

[25] M. Migliorati, L. Palumbo, C. Zannini, N. Biancacci, and V. G. Vaccaro, Resistive wall impedance in elliptical multilayer vacuum chambers, Phys. Rev. Accel. Beams 22, 121001 (2019).

[26] L. Vos, The transverse impedance of a cylindrical pipe with arbitrary surface impedance, CERN, Geneva, Switzerland, Tech. Rep. CERN-AB-2003-005-ABP, 2003, http://cds .cern.ch/record/604828.

[27] A. Koschik, F. Caspers, E. Métral, L. Vos, and B. Zotter, Transverse resistive wall impedance and wake function with "inductive bypass", in 9th European Particle Accelerator Conference (EPAC 2004), 7 2004, http://cds.cern .ch/record/702820.

[28] L. Malina, J. M. Coello de Portugal, J. Dilly, P. Skowroński, R. Toms, and M. Toplis, Performance optimisation of turn-by-turn beam position monitor data harmonic analysis, in 9th International Particle Accelerator Conference, Vancouver, Canada (JACoW, Geneva, 2018), http://cds.cern.ch/record/2672234.

[29] A. W. Chao and M. Tigner, Handbook of Accelerator Physics and Engineering (World Scientific, Singapore, 1999), https://cds.cern.ch/record/384825.

[30] W. R. Inc., Mathematica, Version 12.1, champaign, IL, 2020. https://www.wolfram.com/mathematica. 